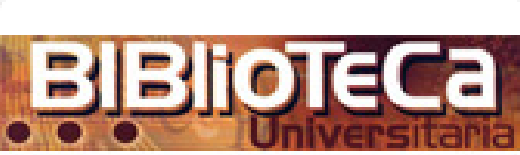

Biblioteca Universitaria

ISSN: 0187-750X

public@dgb.unam.mx

Universidad Nacional Autónoma de México

México

Venteño Jaramillo, Guadalupe; Casas Castillo, Federico

El levantamiento de datos estadísticos en la biblioteca

Biblioteca Universitaria, vol. 16, núm. 1, enero-junio, 2013, pp. 46-53

Universidad Nacional Autónoma de México

Distrito Federal, México

Disponible en: http://www.redalyc.org/articulo.oa?id=28528266005

- Cómo citar el artículo

- Número completo

- Más información del artículo

- Página de la revista en redalyc.org

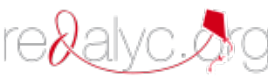

Sistema de Información Científica

Red de Revistas Científicas de América Latina, el Caribe, España y Portugal

Proyecto académico sin fines de lucro, desarrollado bajo la iniciativa de acceso abierto 


\section{El levantamiento de datos estadlísticos en la biblioteca}

\section{The gathering of statistical data in the library}

\section{Guadalupe Venteño Jaramillo*, Federico Casas Castillo*}

\section{RESUInEn}

Se expone la importancia del levantamiento de datos, que al ser procesados sirven para integrar estadísticas en la biblioteca, así como la necesidad de recolectarlos sistematizadamente garantizando su exactitud y legitimidad. También se analiza la importancia del uso de la estadística descriptiva en la biblioteca, tanto en la planeación, organización, administración y evaluación como en la investigación bibliotecológica.

Palabras Clave: Estadística y bibliotecas, información estadística, recolección de datos, dato estadístico.

\section{Abstract}

This paper describes the importance of gathering data that, when processed, are useful to integrate the library statistics, as well as the need to collect them systematically ensuring their accuracy and legitimacy. It also discusses the importance of using descriptive statistics in the library both in planning, organization and management, and their evaluation, and in library research.

KEYwORDS: Statistics and libraries, statistical information, data collection, statistical data

\section{Introducción}

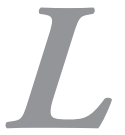

a dinámica de trabajo inmersa en los procesos y servicios de la biblioteca, en la cotidianidad del préstamo, la ordenación de la colección y en la atención a los usuarios, hace que aspectos importantes para identificar el funcionamiento en las bibliotecas, como lo es el levantamiento de datos, pasen desapercibidos.

Al respecto surge necesariamente la siguiente pregunta: ¿qué es el levantamiento de datos?, es la colección o reunión de datos indicados por medio de números y cuadros. ${ }^{1}$ Los datos se recopilan, presentan y describen para integrar estadísticas. De acuerdo a Góngora Cortés, la estadística es "una forma de razonamiento basada en información cuantitativa".

\footnotetext{
* Dirección General de Bibliotecas (DGB). Universidad Nacional Autónoma de México (UnAm). Biblioteca Central, Ciudad Universitaria, 04510 México, D.F. México. Correos electrónicos: ventenov@hotmail.com; casas@hotmail.com

Massa de Gil, Beatriz, Trautman, Ray y Goy, Peter. Diccionario técnico de biblioteconomía, p. 83.

2 Góngora Cortés, José Juan. Estadística descriptiva, p. 14.
} 
La estadística a menudo nos trae a la mente imágenes de números, tablas, volúmenes de cifras relativas a créditos, ingresos, mortalidad, morbilidad y población, entre otros. En la biblioteca la estadística refiere a volúmenes, títulos empleados, egresos, presupuesto, suscripciones, usuarios y personas que laboran en ella; sin embargo, es mucho más que sólo números ordenados y gráficas, es una ciencia tan antigua como la escritura, "y es por sí misma auxiliar de todas las demás ciencias."

En bibliotecología es importante contar con datos estadísticos ya que permiten tener una visión objetiva de lo que sucede y, particularmente, en la planeación facilita la toma de decisiones para resolver situaciones cotidianas para enfrentar el futuro.

La estadística "es la ciencia cuyo objetivo es reunir una información cuantitativa concerniente a individuos, grupos, series de hechos, etcétera, y deducir de ello gracias al análisis de datos unos significados precisos o unas previsiones para el futuro"4. Otra definición indica que "la estadística, en general, es la ciencia que trata de la recopilación, organización, presentación, análisis e interpretación de datos numéricos con el fin de realizar una toma de decisión más efectiva." ${ }^{5}$ La estadística bibliotecaria, de acuerdo a Buonocore, es el conjunto "de datos que tienden a recoger y coordinar grupos de hechos en una biblioteca". ${ }^{6}$

Relacionando estas definiciones con lo que sucede en la biblioteca se puede decir que la estadística sirve para medir entre otros ítems: crecimiento de la colección, usuarios, recursos y también para hacer proyecciones sobre algunos acontecimientos.

La estadística se divide en descriptiva e inferencial. En este documento sólo se hará referencia a la estadística descriptiva, que es la que tradicionalmente se usa

3 Ruiz Muñoz, David. Manual de estadística [en línea]. España:

Eumed-net, 2004, p. 1. <http://www.eumed.net/cursecon/ libreria/drm/drm-estad.pdf>

4 HAYES, Robert M. and BECKER, Joseph. Handbook of data processing for libraries.

5 Idem.

6 Massa de Gil, Beatriz, Trautman, Ray y Goy, Peter, op. cit., p. 83. con propósitos de contar y describir hechos que se presentan en una organización, para mostrar cifras a través de datos numéricos.

La estadística descriptiva se refiere a la tabulación de datos y su presentación en forma gráfica o ilustrativa. El cálculo de medidas descriptivas se hace a través de mediciones cuantificables como son: la media, la moda, la mediana, la desviación estándar, varianza, cuantiles, correlación, regresión y números índices.

Las técnicas estadísticas se aplican de manera amplia en contabilidad, control de calidad, estudios de usuarios, análisis de uso de las colecciones y administración, entre otros. La estadística es utilizada en órganos políticos, cuerpos colegiados, personas que intervienen en la toma de decisiones y desde luego en bibliotecas y centros de información. Se puede decir que la estadística se ha adoptado en todas las ciencias incluyendo la Bibliotecología y las ciencias de la información, entre otras.

El objetivo de este documento es identificar la importancia de realizar un levantamiento de datos ordenado y sistematizado, ya que éstos servirán para integrar estadísticas útiles para la planeación y administración en la biblioteca.

\section{La metodología}

Para la elaboración de este documento se realizó una investigación de tipo documental de gabinete, llevando a cabo búsquedas de información bibliográfica en bases de datos.

\section{Antecedentes de la estadística}

Los comienzos de la estadística han sido hallados en varias culturas y épocas de la humanidad, algunos de los más importantes se dieron en el antiguo Egipto, "cuyos faraones lograron recopilar, hacia el año 3050 antes de Cristo, prolijos datos relativos a la población y la riqueza de ese país". ${ }^{7}$

7 Ruiz Muñoz, David, op. cit., p. 2. 
El registro de riqueza y población se hizo con el objetivo de conocer los recursos disponibles para la construcción de las pirámides, según narra el historiador griego Heródoto; también el faraón egipcio Ramsés ॥ hizo un censo de las tierras con el objeto de verificar un nuevo reparto. ${ }^{8}$

En el Antiguo Testamento, en el Libro de los números se narra que en el antiguo Israel se recogían datos estadísticos obtenidos de dos recuentos de la población hebrea. Por otra parte, el rey David también ordenó contar la población. ${ }^{9}$

Posteriormente, mil años después, con la caída del imperio romano se realizaron muy pocas operaciones estadísticas. En esa época la única que llevaba ordenadamente su relación de las tierras era la Iglesia; esta información era compilada por Pipino el Breve en el año 758 y por Carlomagno en el 762 d.c. En el siglo IX en Francia se realizaron algunos censos parciales de siervos. En Inglaterra, Guillermo el Conquistador recopiló el Domesday Book o libro del Gran Catastro, que para el año 1086 fue el primer compendio estadístico de un documento de la propiedad, extensión y valor, que contenía información sobre las tierras de Inglaterra. Aunque Carlomagno, en Francia, y Guillermo el Conquistador, en Inglaterra, trataron de revivir la técnica romana, los métodos estadísticos permanecieron casi olvidados en la Edad Media.

Durante los siglos xv, xv, y xvI, hombres como Leonardo de Vinci, Nicolás Copérnico, Galileo, Neper, William Harvey, Sir Francis Bacon y René Descartes, hicieron grandes aportaciones al método científico y con ello a la estadística, de tal forma que cuando se crearon los estados nacionales y surgió con fuerza el comercio internacional ya existía un método para la obtención de datos económicos.

En el siglo xVIII y en el xIx, con el uso de la estadística se desarrolló el arte de describir todos los objetos en razón a sus cualidades en un forma lógica a partir de las normas generales a investigar, de las fuentes a re-

8 Historia de la probabilidad y estadística III. Coordinación Jesús Sanos del Cerro.

9 Idem. currir, de los principios a juzgar y de los usos que se daba a los recursos relativos al estado de las naciones.

Desde el siglo pasado y recientemente, los progresos en el campo de la estadística se refieren al desarrollo del cálculo de probabilidades, particularmente en la rama denominada determinismo o relatividad. Se ha demostrado que el determinismo fue reconocido en la física como resultado de las investigaciones atómicas y que este principio se aplica tanto a las ciencias sociales como a las físicas.

La estadística, las estadísticas y el dato estadístico pueden generar cierta confusión, ya que son conceptos y palabras muy similares pero con significados diferentes; a continuación se tratará de hacer una breve descripción de ellos:

a. La estadística es el término que se usa para referirse a toda la información estadística.

b. Las estadísticas son un conjunto de datos y métodos que se utilizan para analizar la información estadística, por ejemplo las estadísticas de población y las estadísticas económicas, entre otras.

c. Lo estadístico se refiere a la medida derivada de una muestra como: las medidas de tendencia central, correlación y regresión.

Tener claro estos conceptos permite hacer mediciones e interpretaciones adecuadas.

\section{La estadística y la biblioteca}

La biblioteca como centro difusor del conocimiento e información debe darse a sí misma información relevante con el fin de organizarla y elaborarla de manera útil y que sirva para la toma de decisiones. ${ }^{10}$

Las bibliotecas actuales se mueven a un ritmo más acelerado y han cambiado debido a las necesidades de los usuarios, por lo que es preciso mantener un control de sus operaciones. Las decisiones de los

10 Manual de biblioteconomía. Editora, Luisa Orera Orera. 
directivos de las bibliotecas pueden tener como sustento un cuidadoso análisis cuantitativo y cualitativo a través del levantamiento de datos estadísticos.

En la planeación bibliotecaria es importante "contar con datos consecutivos y obtenidos por procedimientos uniformes",11 con ellos se podrán construir las estadísticas que servirán para extraer mediciones relevantes. En este contexto, las mediciones permitirán "marcar pautas para la gestión de la colección y obtener información muy valiosa", ${ }^{2}$ útil para tomar decisiones.

Con los datos obtenidos se elaboran estadísticas, las cuales son instrumentos muy importantes para la biblioteca y tres representan los principales tipos: $:^{13}$

- Administrativas: miden al personal y sus actividades con relación a su producción y costos.

- Técnicas: registran pedidos de libros, su proceso físico y menor, el registro de materiales (revistas, artículos).

- Públicas: registran préstamos, servicios, usuarios, uso de la colección, frecuencia de uso de bases de datos, búsquedas bibliográficas, infraestructura, entre otras cuestiones.

En estos tipos de estadísticas es posible describir la generación de datos de todas las áreas de la biblioteca y cuando no se cuenta con toda la información fidedigna se puede caer en el error de dar cifras no confiables, por ello es importante poner atención a esta actividad.

En la biblioteca el uso de la estadística descriptiva considera los datos del promedio de usuarios en un día, el promedio de libros en una semana, el promedio de videos prestados en un año, mapas prestados en un semestre, entre otros.

11 Idem.

12 La biblioteca universitaria: análisis en su entorno híbrido. Editora, Luisa Orera Orera, p. 215.

13 Melnik, Diana y Pereira, María Elina. Bases para la administración de bibliotecas, p. 52-55.

\section{La recolección de datos}

Ésta se hace a través de conteos. El conteo se hace con el propósito de conocer qué sucede en alguna de las áreas, saber cómo crecerá la colección, a qué hora llegan más usuarios a la biblioteca, qué libro es el que más solicitan, cuál es el tema que más se consulta, entre otros muchos objetivos. En realidad en la sociedad actual casi todo lo podríamos contar pero... ¿̇cómo contamos? No siempre se cuenta de la misma forma y es importante que el conteo se haga en las mismas condiciones, en la misma área, a la misma hora, etcétera. ¿Quién cuenta?, la persona responsable de contar debe ser una persona cuidadosa, interesada en saber qué sucede y por qué suceden las cosas.

Para Torgerson la medición es una propiedad que "lleva consigo una asignación de números-objetos como una forma de esa propiedad". ${ }^{14}$ De acuerdo a esta definición es fácil medir propiedades que son observables, como longitudes, tiempos, temperaturas y masas, porque son cuantificables y existen procedimientos específicos para medirlos.

Cuando nos enfrentamos al dilema de medir aspectos no cuantificables, como la inteligencia o la violencia, tenemos dificultades para realizar estas mediciones. El ser humano encontró la forma de llevar a cabo estas mediciones a través de vincular fenómenos observables a conceptos abstractos.

El proceso de medición en ciencias sociales lleva a una consecuencia y una toma de posturas respecto a la posibilidad o formas de realizar las mediciones. En este contexto existe el riesgo de que en toda medida realizada en ciencias sociales exista un nivel de subjetividad. ${ }^{15}$

Para realizar la actividad de computar y levantar los datos es necesario que la persona responsable de ello sea capacitada cuidadosamente y sensibilizada sobre la importancia de esta actividad.

El conteo con fines estadísticos no debe ser sustituido por cifras estimativas o aproximadas porque se falsearía

14 González Blasco, Pedro. Medir en ciencias sociales.

15 Galtung, Johan. Teoría y métodos de la investigación social. 
la información y los resultados, tampoco debe ser tomado como una actividad sin importancia. Se puede caer en la tentación de que con el propósito de aumentar el prestigio de la biblioteca se alteren los datos del número de usuarios asistentes o también -como justificación adicional- para incrementar los recursos: económicos, humanos, documentales, etcétera.

Cuando no se tienen datos confiables (datos duros) se corre el riesgo de tomar decisiones sin fundamento y por lo tanto no se tiene la posibilidad de corregir las deficiencias de un proyecto o un servicio. ${ }^{16}$

Para conocer lo que sucede en un proyecto en particular se recomienda generar reportes que respondan a propósitos específicos y los resultados ofrecerán información para evaluar y gestionar dicho proyecto. ${ }^{17}$

Entonces, los datos son observaciones que se realizan sobre una situación que desconocemos y de la cual deseamos saber ¿qué pasa?, ¿cómo se comporta? y de la que nos interesa conocer ¿̇cómo se comportará en el futuro?, por ejemplo, percibir cuánto crecerá la colección.

\section{El uso y la trascendencia de la estadística en la investigación bibliotecológica.}

La estadística es una herramienta útil en las ciencias sociales que permite planear, administrar, organizar y evaluar. ${ }^{18}$ Uno de los más importantes objetivos es evaluar, básicamente; es comparar los resultados alcanzados con los propósitos establecidos; "evaluar es comparar la productividad o el desempeño con las metas prefijadas. Para ello es necesario tener: a) objetivos y metas claras; b) instrumentos de medición; c) técnicas de fácil aplicación y adecuadas a los propósitos." ${ }^{19}$

\footnotetext{
6 CARAvia Santiago. La biblioteca y su organización, p. 83.

7 Idem.

18 MADRID, Isabel. Evaluación de bibliotecas [en línea]: su necesidad e importancia, p. 103-113.

19 Nocettı, Milton A. Técnicas de avaliaçâo nos serviços de informaçâo: notas preliminares.
}

La estadística es una herramienta que permite hacer abstracciones de la realidad a través del pensamiento lógico-matemático, y por medio del uso de números es posible poder cuantificar los fenómenos que nos rodean. Utilizando recursos matemáticos es factible describir lo que sucede, sus características, lo cual ayuda en la toma de decisiones.

Además, la información estadística en las bibliotecas permite conocer la labor diaria, así como su conducta, y en prospectiva permite visualizar el desempeño y crecimiento de la biblioteca. También, sirve para hacer comparaciones de datos estadísticos por periodos de años, semanas, días, etcétera, lo que ofrece una perspectiva sobre la tendencia en los datos y percibir si hay consistencia entre ellos.

Hace algunos años en México las bibliotecas se encontraban en una estructura organizativa con uno o dos niveles jerárquicos inferiores. Por este motivo la información estadística que se solicitaba se refería sólo a unos cuantos items predeterminados y variables, como número de ejemplares comprados, número de revistas, títulos duplicados, número de fotocopias facilitadas, etcétera. Esta situación ha ido cambiando y actualmente la relación establecida con la biblioteca ocupa una posición en el organigrama cercana a la dirección de la institución o dependencia a la que pertenece, motivo por el cual la información que se requiere conocer demanda mayor precisión.

También, con el avance de las tecnologías y los servicios de información se han ampliado los tipos de datos que se levantan, y con esta situación el tipo de información que se genera es mayor. Las bibliotecas -en general-, necesitan precisar y definir su misión, sus objetivos y los servicios para sus usuarios; desde esta perspectiva no es suficiente, un informe cuantitativo de variables numéricos para saber si la biblioteca cumple o no con los objetivos señalados, ${ }^{20}$ se requiere ir más allá a través de los análisis cualitativos.

La información estadística es el resultado de la recopilación de datos y no refleja totalmente lo que sucede

20 Fuentes, Juan José. Evaluación de bibliotecas y centros de documentación e información, p. 29-30 
en la biblioteca, ${ }^{21}$ después del levantamiento de datos se debe ir integrando información adicional. Algunos de los resultados de las cifras son las gráficas que nos indican el estatus en el que se encuentra una variable en particular. Los procedimientos estadísticos por sí solos sirven para planificar recursos humanos, materiales, instalaciones; reflejan el aspecto numérico cuantitativo de una situación pero no explican otros elementos necesarios para una evaluación de conjunto.

En este sentido, la estadística sigue siendo necesaria -por ejemplo- para conocer el nivel de gasto en la colección por habitante o por usuario, el número de préstamos o el número de metros cuadrados de las instalaciones, el espacio del cual hace uso el lector de las publicaciones periódicas, el grado de satisfacción de los usuarios, pero debe de ir apoyada con más información construida a partir de datos cualitativos.

Recientemente, debido a las restricciones presupuestales, las bibliotecas se han interesado cada vez más en las técnicas que pueden utilizarse para evaluar los servicios que se proporcionan. Las bibliotecas universitarias han sido las más interesadas en realizar diversos tipos de mediciones, algunas de ellas que han comenzado a trabajar este aspecto y a aplicar indicadores específicos son la Universidad Anáhuac México Sur y la Dirección General de Bibliotecas de la UnAM, entre otras.

\section{Limitaciones de la estadística}

Es necesario tener especial cuidado en la obtención de los datos estadísticos, por lo que es ineludible ha-

21 Ibid., p. 30.

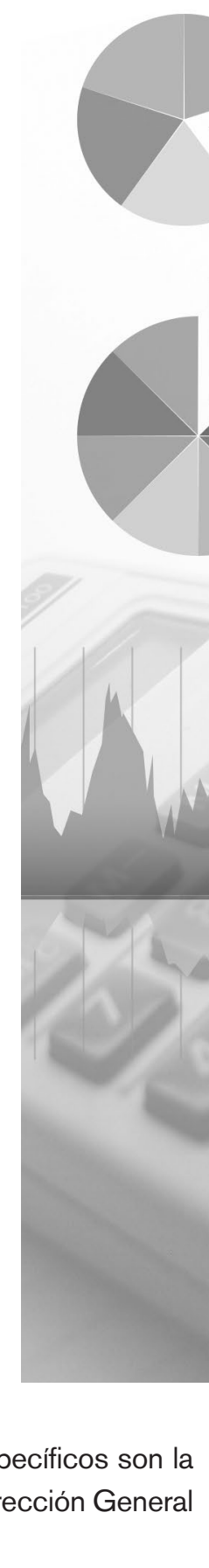

cer una planeación para su levantamiento. Posteriormente, y una vez que se han recogido los datos, es conveniente analizarlos y tratar de ser lo más objetivo posible para poder hacer una adecuada interpretación y análisis de los mismos.

Los datos por sí mismos no revelan mayor información, por ello se conforman estadísticas; éstas permitirán agruparlos relacionándolos con variables que ofrezcan mayor información de un hecho para hacer un análisis. 
La superficialidad o el abuso de la estadística por algunas personas ha hecho que parezca más una cómplice que una colaboradora, es por ello que se dice que los datos reflejan lo que uno quiere, porque no siempre corresponden a la realidad. Lo anterior sucede cuando no se tienen planteamientos teóricos y metodológicos sólidos.

El hecho de que se prioricen números tiende a hacer que la gente considere que los datos recabados son mucho más objetivos y definitorios de lo que realmente son. Hay quienes sostienen que si uno no puede cuantificar un problema o una solución es porque no la entiende en su totalidad. Esto es cierto, pero también lo es que uno puede cuantificar un problema o una solución sin entenderla realmente. Es entonces cuando se debe ser muy cauteloso en la forma de emplear las técnicas cuantitativas que ofrecen modelos auxiliares para interpretar una realidad, pero no son la respuesta. ${ }^{22}$

Para analizar la información estadística los expertos recomiendan: ${ }^{23}$

- En primer lugar, debe existir un objetivo que debe maximizarse o minimizarse en términos de valor, y el objetivo necesita expresarse de alguna manera cuantificable: dinero, tiempo o cantidad.

- En segundo lugar, las variables que se midan deben tener un efecto sobre el resultado. En una biblioteca, en el departamento de circulación, las tareas realizadas son de diferente tipo, por ello es importante establecer relaciones entre las actividades que se realizan y el levantamiento de los datos.

- En tercer lugar, deben existir restricciones a la interrelación de las variables; sin estas restricciones la programación no sería necesaria porque el objetivo en su totalidad podría encararse de otra manera. ${ }^{24}$

22 Evans, G. Edward. Técnicas de administración para bibliotecarios

${ }_{23}$ HAYES, Robert M. and BECKER, Joseph, op. cit.

${ }^{24}$ Idem

\section{Recomendaciones}

- Planear primeramente qué es lo que se quiere medir, para qué se mide, y para qué se va a utilizar.

- Determinar qué forma tiene el instrumento que se genera para levantar los datos, tales como formularios, cuestionarios, encuestas, entrevistas y otras observaciones.

- Determinar los tiempos para el levantamiento de datos; puede ser de manera continua (diario, mensual, semanal, etcétera).

- Establecer la manera de representar los datos, cómo gustaría que se presentaran; puede ser por medio de gráficos, tablas, cuadros de resúmenes o una mezcla de estos tres.

- Utilizar de preferencia software que permita facilitar la captura de datos y su recuperación.

- Una vez obtenidos y procesados los datos estadísticos se pueden analizar y calcular indicadores que permitan examinar datos e información para la toma de decisiones.

- Llevar a cabo un levantamiento estadístico de datos en todas las áreas generando reportes específicos para cada una de ellas.

- Garantizar que el personal que cotidianamente levanta los datos (a través de cuestionarios o encuestas) sea el mismo, con el propósito de dar continuidad al proceso.

\section{Conclusiones}

Es importante llevar a cabo el levantamiento de datos de una forma ordenada y sistematizada que permita generar en un período determinado estadísticas sobre diferentes áreas de la biblioteca. El conjunto de estadísticas, así como la utilización de las medidas de tendencia central, pueden servir para los procesos de planeación, administración, organización y evaluación en la biblioteca. cos 


\section{Obras Consultadas}

La biblioteca universitaria: análisis en su entorno híbrido. Editora, Luisa Orera Orera. Madrid: Síntesis, 2005,477 p.

Caravia, Santiago. La biblioteca y su organización. España: Trea, 1995. 198 p.

Evans, G. Edward. Técnicas de administración para bibliotecarios. México: unAM, 1980, 317 p.

Fuentes, Juan José. Evaluación de bibliotecas y centros de documentación e información. España: Ediciones Trea, 1999. 237 p.

Galtung, Johan. Teoría y métodos de la investigación social. Buenos Aires: Eudeba, 1966. V.

Góngora Cortés, José Juan. Estadística descriptiva. México: Trillas, 1999. 365 p.

González Blasco, Pedro. Medir en ciencias sociales. En: El análisis de la realidad social: métodos y técnicas de investigación. Compilación de Manuel García Ferrando, Jesús Ibáñez y Francisco Alvira. Madrid: Alizanza, 2000. 682 p.

Hayes, Robert M. and Becker, Joseph. Handbook of data processing for libraries. usa: John Wiley \& Sons, 1974.

Historia de la probabilidad y estadística III. Coordinación Jesús Sanos del Cerro. Madrid: Delta, 2006.

MAdrid, Isabel. Evaluación de bibliotecas [en línea]: su necesidad e importancia. Información, cultura y sociedad, enero-junio 2002, <http://www.scielo.org.ar/scielo.php?script=sci_arttext\&pid=S1851-17402002000100008> [Consulta: octubre 2012].

Manual de biblioteconomía. Editora, Luisa Orera Orera. Madrid: Síntesis, 1996. 509 p.

Massa de Gil, Beatriz, Trautman, Ray y Goy, Peter. Diccionario técnico de biblioteconomía. México: Centro Regional de Ayuda Técnica, 1984. 387 p.

Melnik, Diana y Pereira, María Elina. Bases para la administración de bibliotecas. Buenos Aires: Alfagrama, 2005.205 p.

Nocettr, Milton A. Técnicas de avaliaçâo nos serviços de informaçâo: notas preliminares. Brasilia: EMBRAPA., 1978.

Ruiz Muñoz, David. Manual de estadística [en línea]. España: Eumed-net, 2004. 91 p. < http://www.eumed.net/ cursecon/libreria/drm/drm-estad.pdf> [Consulta: octubre 2012] 